\title{
Fracture behaviour of unmodified and rubber-modified epoxies under hydrostatic pressure
}

\author{
D. LI, A. F. YEE*, I. -W. CHEN \\ Department of Materials Science and Engineering, The University of Michigan, \\ Ann Arbor, MI 48109, USA
}

S. - C. CHANG ${ }^{\dagger}$

Department of Materials Science and Engineering, Tsing Hua University, Hsinchu, Taiwan

K. TAKAHASHI

Research Institute for Applied Mechanics, Kyushu University, Kasuga, Japan

The fracture toughness and uniaxial tensile yield strengths of unmodified and CTBN-rubbermodified epoxies were measured under hydrostatic pressure. The purpose of these experiments was to learn how suppressing cavitation in rubber particles affects the deformation mechanisms and the fracture toughness of rubber-modified epoxy. It was found that the cavitation of CTBN-rubber could be suppressed at a relatively low pressure (between 30 and $38 \mathrm{MPa}$ ). With cavitation suppressed, the rubber particles are unable to induce massive shearyielding in the epoxy matrix, and the fracture toughness of the rubber-modified epoxy is no higher than that of the unmodified epoxy in the pressure range studied. Unmodified epoxy shows a brittle-to-ductile transition in fracture toughness test. The reason for this transition is the postponement of the cracking process by applied pressure.

\section{Introduction}

The toughening mechanism of CTBN-rubber-modified epoxy has been studied by many researchers [1-8]. Yee and co-workers found that the ability of CTBN-rubber to toughen epoxy is closely related to CTBN-rubber cavitation $[3,4,7,8]$. Their previous work $[3,8]$ shows that the physical reason why rubber cavitation is essential to toughening is that upon cavitation the rubber particles cause constraint relief within the surrounding matrix. Owing to this effect, the matrix material can shear deform more easily because it is in a less constrained condition. If the concentration of cavitated rubber particles is sufficiently high, the plane stress state can actually be reached [8]. The plane stress state is favourable for plastic deformation, which allows the matrix epoxy to absorb more energy. The cavitation mechanism is therefore effective only in materials that are capable of plastic deformation in the plane stress state, but which undergo brittle failure in the plane strain state, i.e. in the multiaxial tensile stress state.

The above conclusions were drawn from microscopic analysis of specimens containing rubber particles capable of cavitating, in which subcritical crack growth or fracture had occurred, and from macro- scopic examinations such as tensile dilatometry and other tests described in detail elsewhere [8]. To establish fully the importance of cavitation in the toughening process and to verify the proposed toughening mechanism, it is necessary to determine what happens if no cavitation is allowed to occur.

Two approaches can be used to prevent rubber cavitation from occurring: (1) chemical modification of the rubber to increase its cavitation resistance, and (2) physical suppression of cavitation, which is a volume dilatation process, by applying hydrostatic pressure during mechanical testing. Because it is very difficult to change only the cavitation resistance of a rubber without affecting its bonding with and dispersion in the matrix epoxy, and as the physical suppression of cavitation neither changes the size and dispersion of the rubber particle, nor the bonding of rubber to the matrix, we have chosen to use the second approach.

The study of the mechanical properties of materials under pressure dates back at least to Nadai [9] and Bridgman [10]; however, the application of this technique to the study of synthetic polymers is much more recent. Under pressure, a transition in the fracture mode from brittle to ductile has been reported

* Author to whom all correspondence should be addressed.

${ }^{\dagger}$ Work performed while on a sabbatical leave at the University of Michigan. 
for amorphous polymers including polystyrene (PS) [11-14], poly(methyl methacrylate) (PMMA) [15-17], poly(vinyl chloride) (PVC) [18] and polyimide (PI) [19]. Some of these results were found to be affected by the pressure-transmitting fluid used, especially in the low-pressure range $[11,15,20]$. By using Teflon tape and a transparent silicone rubber to exclude the pressure-transmitting fluid from coming into contact with the polymer surface, brittle to ductile transitions were found to occur under sufficiently high pressure. At room temperature, the brittle-to-ductile transition pressure is $45 \mathrm{MPa}$ for PS [11], 25 $\mathrm{MPa}$ for PMMA [15], and as low as $20 \mathrm{MPa}$ for PVC [18]. This brittle-to-ductile transition was suggested by Baer and co-workers $[14,15,18]$ to be due to different pressure dependences of competing microdeformation processes of crazing and shear-banding. The crazing process, which involves volume dilatation, is suppressed by hydrostatic pressure. This leaves shearyielding as the sole remaining mechanism. Therefore, the fracture mode changes from brittle to ductile.

A number of researchers have also studied the deformation and fracture behaviour of rubber-modified polymers such as HIPS and found that they behave like unmodified PS when the pressure is sufficiently high $[14,21]$. By using a sealing technique to isolate the sample surface from the pressure-transmitting fluid, Trent et al. [21] showed that the stresswhitening (multiple-crazing) usually found in HIPS was suppressed at relatively low pressures and the material failed in a ductile manner over the entire pressure range studied (up to $700 \mathrm{MPa}$ ). The yield and fracture stresses of HIPS increased with increasing hydrostatic pressure, but an initial reduction in the elongation to fracture occurred until a minimum was reached at around $45 \mathrm{MPa}$; thereafter, the elongation increased with pressure. This minimum corresponded to the disappearance of stress-whitening. It was concluded that the deformation and fracture mode changed from multiple-crazing in the pressure range from one atmosphere to $12 \mathrm{MPa}(0.12 \mathrm{kbar})$ to mixed crazing and shear-banding (necking) in the pressure range $12-40 \mathrm{MPa}(0.12-0.4 \mathrm{kbar})$, and to shearbanding only at pressures above $40 \mathrm{MPa}(0.4 \mathrm{kbar})$.

Most of the published work on the hydrostatic pressure effect was carried out in the uniaxial tensile mode on unnotched specimens, which gives only tensile strength and ductility information. In a limited number of papers by Duckett and co-workers [22-24], the fracture toughness of polyethylene (PE) was evaluated under hydrostatic pressure. They found that at sufficiently high pressures both $\mathrm{PE}$ and a tough copolymer of PE failed in a brittle manner when a surface notch was exposed to a suitable pressuretransmitting fluid. The increase of yield stress with pressure was offered as the reason for the ductilebrittle transition. An extrapolation method was proposed to estimate the critical stress intensity factor at atmospheric pressure based on the toughness of PE under pressure. They suggested that this provides a means of short-term fracture testing of very ductile polymers that would otherwise be accessible only by low-temperature or long-term testing [24].
The work reviewed above, although not comprehensive, illustrates that the superposition of hydrostatic pressure during mechanical testing provides a useful approach for evaluating the behaviour of polymer materials in complicated stress states that cannot be achieved by experiments conducted at atmospheric pressure. The superimposed hydrostatic pressure generally causes an increase in the yield stress of polymers. Polymers whose deformation involves volume dilatation such as crazing will experience suppression of such processes due to the superimposed hydrostatic pressure. For this reason, the hydrostatic pressure will certainly affect cavitation, which is also a volume dilatation process. In the research reported here, we tested the fracture toughness and uniaxial tensile yield strengths of unmodified and CTBN-rubber-modified epoxies under hydrostatic pressure and followed this by analysing the morphology by optical microscopy and SEM. The purpose of these experiments was to learn how suppression of cavitation in rubber particles affects the deformation mechanisms and the fracture toughness of rubber-modified epoxy. These experiments also provided a direct test for the contention that epoxies are toughened by virtue of the low modulus of the rubber particles and that the cavitation is simply an incidental or even deleterious mechanism $[25,26]$.

\section{Experimental procedure}

\subsection{Materials}

The materials used in this research consisted of Epoxy DER $^{\circledR} 331$ and HYCAR ${ }^{\circledR}$ CTBN $1300 \times 8$ rubber. Epoxy DER 331 is a liquid diglycidyl ether of bisphenol-A (DGEBA) with an epoxy equivalent weight of $186-192(\mathrm{~g})$ and is produced by the Dow Chemical Co. The CTBN $1300 \times 8$ rubber is a carboxyl-terminated acrylonitrile-butadiene copolymer and is produced by the B. F. Goodrich Co. Details concerning this rubber can be found elsewhere [27]. The curing agent was piperidine purchased from the Aldrich Chemical Co. and used as-received.

The preparation of the rubber-modified epoxy is similar to that previously described by Yee and Pearson [3]. Briefly, liquid rubber and epoxy of the desired ratio were mixed and allowed to pre-react at $80^{\circ} \mathrm{C}$ for $1 \mathrm{~h}$. The curing agent piperidine was then added and this pre-reacted mixture was poured into a mould which had been pre-heated to $120^{\circ} \mathrm{C}$. The mixture was allowed to react at that temperature for $18 \mathrm{~h}$, resulting in a cast plaque of $250 \mathrm{~mm} \times 200 \mathrm{~mm} \times 3 \mathrm{~mm}$ (or $6 \mathrm{~mm}$ ). Unmodified epoxy was prepared using the same curing conditions, but without the pre-reaction step. The glass transition temperature determined by differential scanning calorimetry (DSC) at a testing rate of $10^{\circ} \mathrm{C} \mathrm{min}^{-1}$ was $92^{\circ} \mathrm{C}$ for unmodified epoxy and $86^{\circ} \mathrm{C}$ for the 10 p.h.r. CTBN-rubber-modified epoxy. The mechanical properties of these materials are listed in Table I.

\subsection{Mechanical testing methods}

The experimental apparatus used in this research is shown in Fig. 1 and was previously described in detail 
TABLE I Mechanical properties of unmodified and 10 p.h.r. rubber-modified epoxies

\begin{tabular}{llll}
\hline Materials & $\begin{array}{l}E \\
(\mathrm{GPa})\end{array}$ & $\begin{array}{l}\sigma_{\mathrm{y}} \\
(\mathrm{MPa})\end{array}$ & $\begin{array}{l}K_{\mathrm{IC}}^{\mathrm{a}} \\
\left(\mathrm{MPa} \mathrm{m}^{\mathbf{1} 2}\right)\end{array}$ \\
\hline $\begin{array}{l}\text { Epoxy: DER 331 } \\
\text { Epoxy/CTBN: DER331/Pip/ }\end{array}$ & 2.94 & 74 & 0.85 \\
CTBN-8 10 p.h.r. & 2.6 & 63 & 2.1 \\
\hline
\end{tabular}

${ }^{a}$ Determined by using single-edge-notched type specimens in threepoint bending tests.

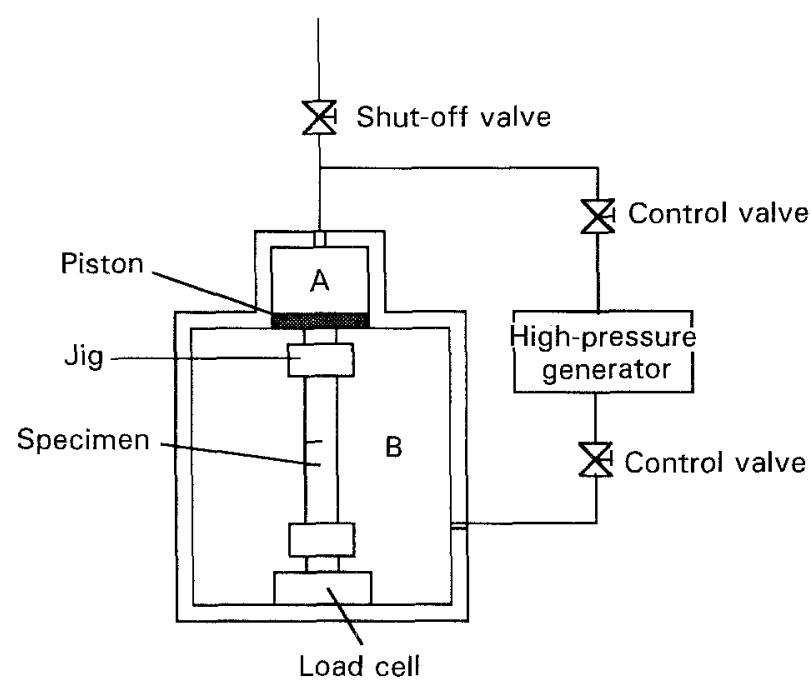

Figure I Schematic illustration of the high-pressure testing apparatus.

by Takahashi and Ogihara [28]. It consists of two concentric cylinders connected by a piston. The larger cylinder is the specimen chamber, which has an inner diameter of $60 \mathrm{~mm}$ and can withstand pressures up to $250 \mathrm{MPa}$. The specimen to be tested is mounted with one end connected to the bottom of the larger cylinder and the other to the piston. Before the tensile test, the two cylinders are filled with a pressure-transmitting fluid at the same pressure. To begin the test, the shutoff valve on the smaller cylinder is opened. Thus, the pressure difference between the two cylinders drives the piston outwards, which loads and eventually fractures the specimen. The load is recorded by an electrical resistance strain gauge load cell built inside the large cylinder. Because of the outward motion of the piston, the total volume of the specimen chamber increases and, as a result, the pressure drops. Special care was taken in the design of the apparatus to make the diameter of the piston relatively small compared with the sample chamber so that the pressure drop is small. The average value of pressures before and after the testing was taken as the pressure imposed on the specimen.

Specimens for tensile testing were machined directly from the cast plaque mentioned earlier. The thickness of the plaque and the specimens was $3.0 \mathrm{~mm}$. The tensile specimens were of the conventional dumb-bell shape. The overall specimen length was $65 \mathrm{~mm}$. The parallel gauge section was reduced from $12.5 \mathrm{~mm}$ at the ends to $10 \mathrm{~mm}$. Specimens were machined by a milling machine. The specimen gauge section was polished with wet no. 600 sand paper, followed by wet no. 1500 sand paper, then with a cotton cloth soaked with water and $5 \mu \mathrm{m}$ polishing powder, and finally finished with a cotton cloth soaked with water and $1.0 \mu \mathrm{m}$ polishing powder. This procedure was employed to minimize the effect of surface flaws on the mechanical behaviour.

Single-edge notched specimens were tested in tension to determine the fracture toughness of both unmodified and rubber-modified epoxies. The specimens were $118 \mathrm{~mm}$ long, $21 \mathrm{~mm}$ wide and $3 \mathrm{~mm}$ thick. Sharp edge cracks were introduced into the specimens in the following way: first, a saw-cut notch $2.5 \mathrm{~mm}$ in depth was made and then a sharp crack was initiated by tapping a razor blade that had been chilled in liquid nitrogen into the saw cut with a hammer. This crack-initiation procedure results in very sharp cracks, and has been discussed in detail previously $[3,4]$.

Owing to the design of the apparatus, the hydrostatic pressure also serves to produce traction on the specimen so the rate of deformation depends on the pressure, the friction of the piston against the wall of the small cylinder, and the resistance of the specimen to deformation. Furthermore, the load-time curve can be obtained but not the strain-time relationship. The strain rate can be estimated by assuming that the strain at yield is not pressure sensitive. At atmospheric pressure the strain at yield is $4.5 \%$. The average strain rate is then estimated by dividing the yield strain by the time to yield. This gives a strain rate of $4 \mathrm{~s}^{-1}$ under a hydrostatic pressure of $80 \mathrm{MPa}$, which is two to three orders of magnitude higher than the conventional tensile strain rate $\left(0.01-0.001 \mathrm{~s}^{-1}\right)$ used by most researchers.

The pressure-transmitting fluid used in this research was kerosene oil. Chemical resistance data [29] show that cross-linked epoxy is very stable in such a fluid. The effect of this fluid on the mechanical behaviour of epoxy was determined and the results are presented and discussed in the next section.

\subsection{Microscopy analysis}

Optical microscopy was used to examine the subsurface damage of fractured specimens. Thin sections perpendicular to the fracture surface and parallel to crack propagation direction were obtained by petrographic thinning techniques. This included embedding the damage zone portion of a specimen in a clear mounting epoxy resin, then reducing the thickness by a combination of cutting and polishing until a final thickness of $25 \mu \mathrm{m}$ or less was reached. The finished sections were examined using a Nikon research microscope, with or without crossed polarizers, for observation of deformations such as plastic shear-induced birefringence and cavitation.

SEM was used to examine the fracture surfaces of both unmodified and CTBN-rubber-modified epoxies. SEM was also used to reveal subsurface rubber deformation. In this case, a section perpendicular to the fracture surface and parallel to the direction of crack propagation was made by microtoming with a diamond knife on a Reichert-Jung Microtome. Both 
fractured and microtomed surfaces were coated with a thin film of gold by sputtering before SEM examination. The instrument used was a Hitachi S-800 scanning electron microscope operating at $4 \mathrm{kV}$.

\section{Results and discussion}

\subsection{Preliminary experiments}

To examine the possible influence of the pressuretransmitting fluid on the deformation and fracture behaviour of unmodified and rubber-modified epoxies, we performed the following experiments: (1) specimen weight check before and after pressurization, (2) comparative fracture toughness tests made at atmospheric pressure in air and in the pressure-transmitting fluid, (3) tensile and fracture toughness tests of specimens with different geometry, and (4) fracture toughness test of specimens soaked under pressure for different periods of time.

The results were as follows: (1) The weight gain of specimens tested after pressurization up to $100 \mathrm{MPa}$ was found to be less than $0.05 \%$. (2) The comparative fracture toughness test results are listed in Table II, and they show that, at atmospheric pressure, the fracture toughness results of both unmodified and CTBN-rubber-modified epoxies in the kerosene oil were within $5 \%$ of those obtained in the air. The standard deviation of the fracture toughness of unmodified epoxy is $0.07 \mathrm{MPam}^{1 / 2}$, or $8 \%$. Thus, the difference is insignificant. These facts confirm that the pressure-transmitting fluid does not affect the fracture toughness at atmospheric pressure. (3) The tensile tests show that kerosene oil is not an environmental crazing/cracking agent for epoxy, even under high pressure. If that were the case, it would have caused premature failure of the epoxy. In fact, both materials became stronger and tougher under pressure. On the other hand, the fracture toughness tests also show that kerosene oil is not a plasticizer for epoxy, either. If that were the case, the toughness and the observed plastic zone would have been influenced by the amount of kerosene oil diffusing into the surface of the specimen under pressure. Because an immersed specimen is in total contact with the fluid, including the crack tip (Fig. 2a), the plasticized zone, if it forms, would be in the shape illustrated in Fig. $2 b$ and the resulting plastic deformation zone would be in the shape illustrated in Fig. 2b. However, the experimental results indicated that a plastic zone existed only in front of the pre-crack and no plastic deformation was observed at the side surfaces (Fig. 2c). This eliminates the possibility of the existence of a static diffusion-controlled plasticized zone. Furthermore, the plastic zone is more than $3 \mathrm{~mm}$ long. Because the actual tensile fracture

TABLE II Fracture toughness of unmodified and 10 p.h.r. CTBN-rubber-modified epoxies under room atmosphere

\begin{tabular}{lll}
\hline & $K_{\mathrm{IC}} \pm \sigma_{\mathbf{n}}\left(\mathrm{MPa} \mathrm{m}^{1 / 2}\right)$ \\
\cline { 2 - 3 } & In air & In kerosene oil \\
\hline Unmodified epoxy & $0.81 \pm 0.07$ & $0.84 \pm 0.07$ \\
Epoxy/CTBN-8 10 p.h.r. & $2.05 \pm 0.05$ & $2.10 \pm 0.06$ \\
\hline
\end{tabular}

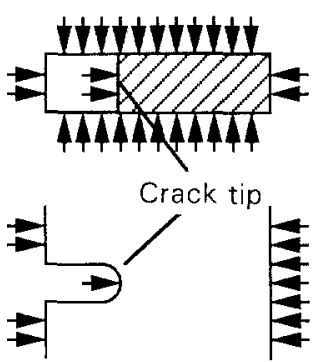

(a)

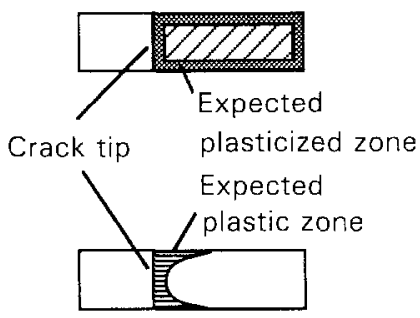

(b)

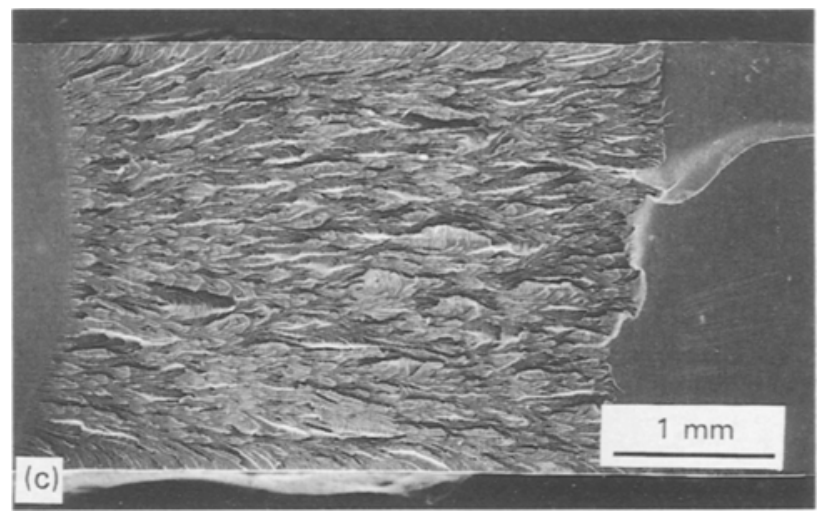

Figure 2 Schematic illustration of the plastic zone expected if kerosene oil is a plasticizer of epoxy: (a) positions in contact with kerosene oil; (b) expected plasticized zones for a single-edge notched specimen; (c) scanning electron micrograph of the fracture surface plastic zone of a single-edge notched specimen.

takes place in less than $2 \mathrm{~ms}$, it is impossible for stressassisted diffusion of the kerosene to take place over this distance in such a short time. These results demonstrate that plasticization of the epoxy by the pressurizing fluid did not occur to any significant extent. (4) Longer soaking time under pressure (30 min compared with $1.5 \mathrm{~min}$ in a regular test) was tried to see if fracture toughness increases with soaking time, but the result was negative.

In conclusion, kerosene oil is neither an environmental cracking agent nor a plasticizer for the epoxy. Both unmodified and CTBN-rubber-modified epoxies are very stable in kerosene oil, and this may be due to their cross-linked structure. The results we observed in the tensile and fracture tests are due to the pressurization effect only.

\subsection{Suppression of cavitation by applied hydrostatic pressure}

The suppression of cavitation by the applied hydrostatic pressure is first evidenced by the disappearance of the stress-whitened zone in specimens tested in simple tension. A photograph of a series of 10 p.h.r. CTBN-rubber-modified epoxy specimens tested in uniaxial tension under different levels of superimposed pressures is shown in Fig. 3. For specimens tested under a pressure of $30 \mathrm{MPa}$ or below, a very distinctive stress-whitened zone due to the presence of numerous cavities in the material can be seen, and the extent of stress-whitening decreases gradually as the applied pressure is raised. When hydrostatic pressure is raised to $38 \mathrm{MPa}$, the stress-whitening virtually disappears. This phenomenon can also be observed in notched tensile tests, and $38 \mathrm{MPa}$ is the minimum hydrostatic 


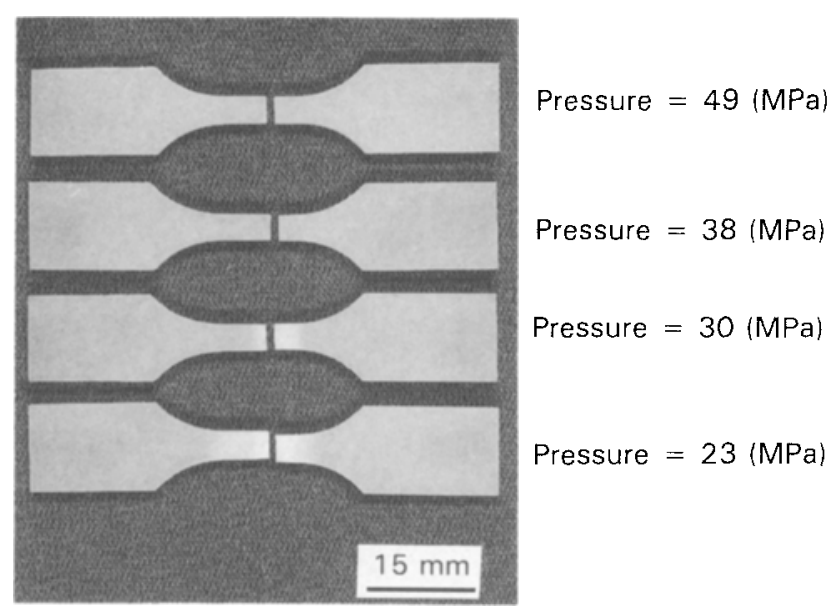

Figure 3 CTBN-rubber-modified epoxy specimens tested at different pressures to demonstrate the disappearance of stress-whitening (rubber cavitation).

pressure at which stress-whitening is eliminated. In our previous work $[3,4,8]$, we demonstrated, by a combination of optical and scanning electron microscopy, that cavitation of CTBN-rubber is the cause of stress-whitening. For the present research we used the same techniques to demonstrate that cavitation is suppressed when the applied hydrostatic pressure is $38 \mathrm{MPa}$ or higher. The observation of stress-whitening depends on the occurrence of two sequential events: rubber cavitation or debonding, and the growth of the resultant voids into larger ones by plastic shear. Because the yield strength of the epoxy is not very sensitive to pressure (see Fig. 6 below), we conclude that the disappearance of stress-whitening is due to the suppression of cavitation of the rubber, and not because the shear plasticity occurs with significantly more difficulty.

SEM was used first to examine the surface of CTBN-rubber-modified epoxy specimens fractured under different pressures, and the results are shown in Fig. 4. Fig. 4a and $\mathrm{c}$ show the fracture surfaces of 10 p.h.r. CTBN-rubber-modified epoxy tested at atmospheric pressure and at a pressure of $80 \mathrm{MPa}$, respectively. Three regions can be seen: (I) pre-crack; (II) slow crack growth and (III) unstable fracture. Although the two specimens had almost identical geometries and crack lengths, the size of the slow crack growth region for the pressurized specimen is substantially larger than that of the one tested at atmospheric pressure. The fracture surface of the pressurized specimen is much rougher than the other. These observations will be further discussed in a later section. Fig. $4 b$ and $d$ show the same slow crack growth regions as in (a) and (c), respectively, but at higher magnification. The fracture surface of the specimen tested at atmospheric pressure (b), reveals that almost all the rubber particles had cavitated prior to rupture. On the fracture surface of the specimen tested under a pressure of $80 \mathrm{MPa}(\mathrm{d})$, most rubber particles did not form cavities prior to rupture. We note that cavitation must be followed by the subsequent growth of the resulting void, i.e. by plastic flow of the surrounding matrix, in order for the cavities to be visible. Otherwise cavitation may not be apparent. Because of the cavitation and subsequent dilatation, the rubber particles in micrograph (b) appear larger and deeper than those in micrograph (d). We note also that some

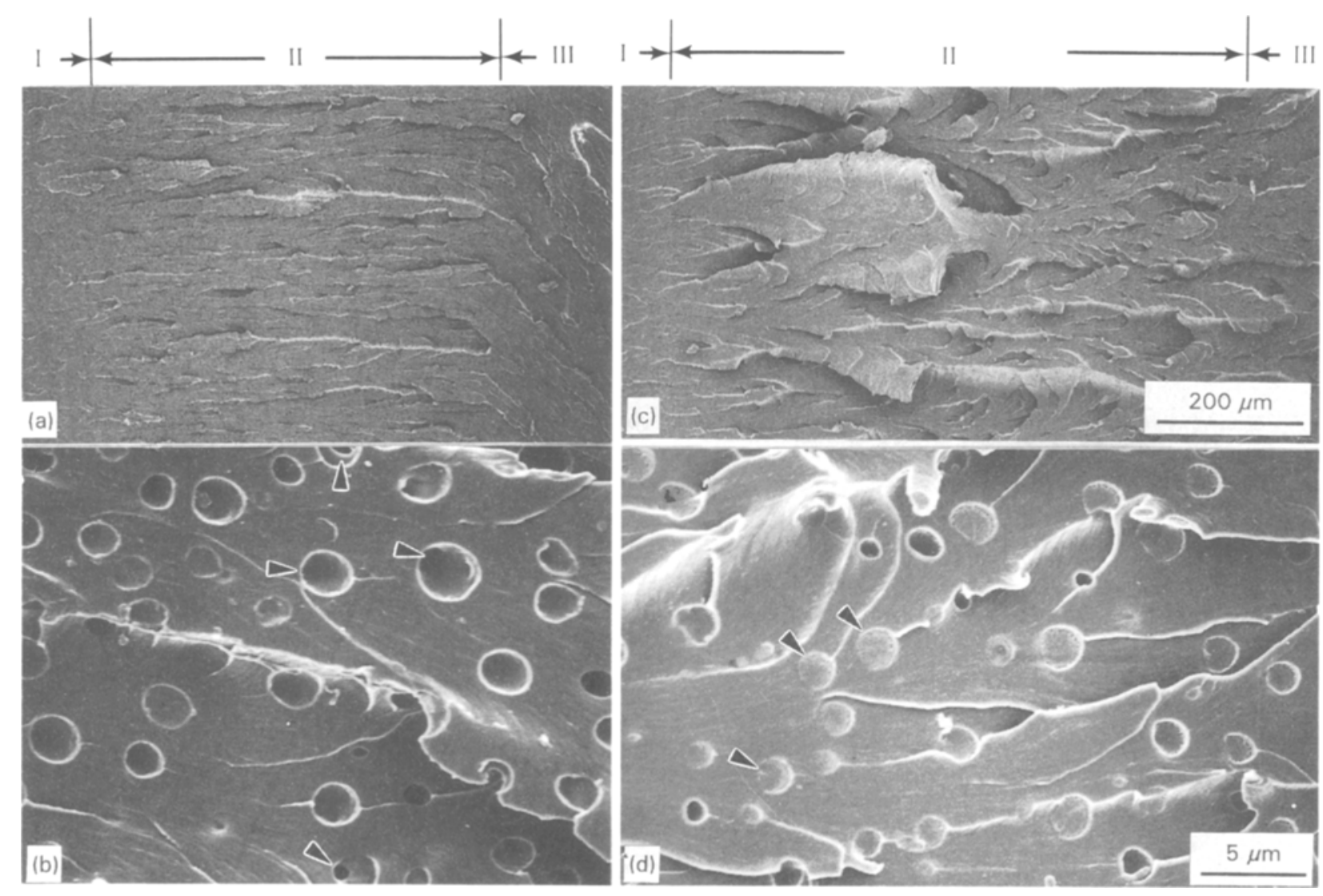

Figure 4 Scanning electron micrographs of the fracture surfaces of CTBN-rubber modified epoxy specimens tested at different pressures: (a, b) atmospheric pressure; (c, d) $80 \mathrm{MPa}$. 
rubber particles in Fig. 4d exhibit evidence for incipient debonding by the formation of microscopic cavities at the interface. Such a phenomenon is to be expected when the cavitation resistance of the rubber is increased, in this case by the superimposed pressure.

Suppression of cavitation of rubber by applied hydrostatic pressure is not just a surface phenomenon. Subsurface cavitation is also affected by pressure. SEM of microtomed sections perpendicular to the fracture surface and parallel to the direction of crack propagation was conducted, and the results are depicted in Fig. 5. Fig. 5a gives the relationship of the microtomed section to the fracture surface. Fig. 5b shows the damage zone of a 10 p.h.r. CTBN-rubbermodified epoxy specimen tested at atmospheric pressure. It shows that cavitation of rubber particles accompanied by plastic dilatation of the surrounding epoxy occurs both on and below the fracture surface in the damage zone. Fig. $5 \mathrm{c}$ is from a specimen of the same material but fractured under a hydrostatic pressure of $80 \mathrm{MPa}$. It indicates that no rubber cavitation had occurred, either on or below the fracture surface. Consequently, no plastic dilatation had occurred.

In summary, the cavitation of CTBN-rubber was suppressed at a relatively low pressure. For the current material system, the minimum hydrostatic pressure required to eliminate rubber cavitation is between 30 and $38 \mathrm{MPa}$, and is not precisely determined due to the friction in the experimental apparatus. This minimum pressure is related to the critical cavitation resistance of the CTBN-rubber and is well below the tensile yield stress of unmodified and rubber-modified epoxies at atmospheric pressure.

\subsection{The tensile yield and fracture of unmodified and rubber-modified epoxies under pressure}

Over the entire pressure range studied, both unmodified and CTBN-rubber-modified-epoxies yield in tension, but fracture shortly after the peak load is reached. The yield stresses $\sigma_{y}$ of both unmodified and CTBN-rubber-modified epoxies increase with the superimposed hydrostatic pressure. These results are plotted versus the average pressure in Fig. 6. We made no attempt to fit these results to obtain a pressuredependent coefficient of yield stress [30] because the strain rate was not constant in our tests.

An important point to note is that the strain rate in this test is approximately $4 \mathrm{~s}^{-1}$. At such a high strain rate and at atmospheric pressure, these epoxy resins fail before the yield point can be reached. However, with a superimposed hydrostatic pressure of $22 \mathrm{MPa}$ or higher, both materials fail after reaching the yield point at a similar strain rate. This change in failure mode shows that there is a strong effect of the superimposed hydrostatic pressure on fracture. The reason may be that the superimposed hydrostatic pressure reduces the mean stress and therefore suppresses the cracking process, because the local mean stress concentration has been shown to be the cause of cracking in epoxies [8] and other polymer materials [31]. Narisawa and Ishikawa [32] also showed that by
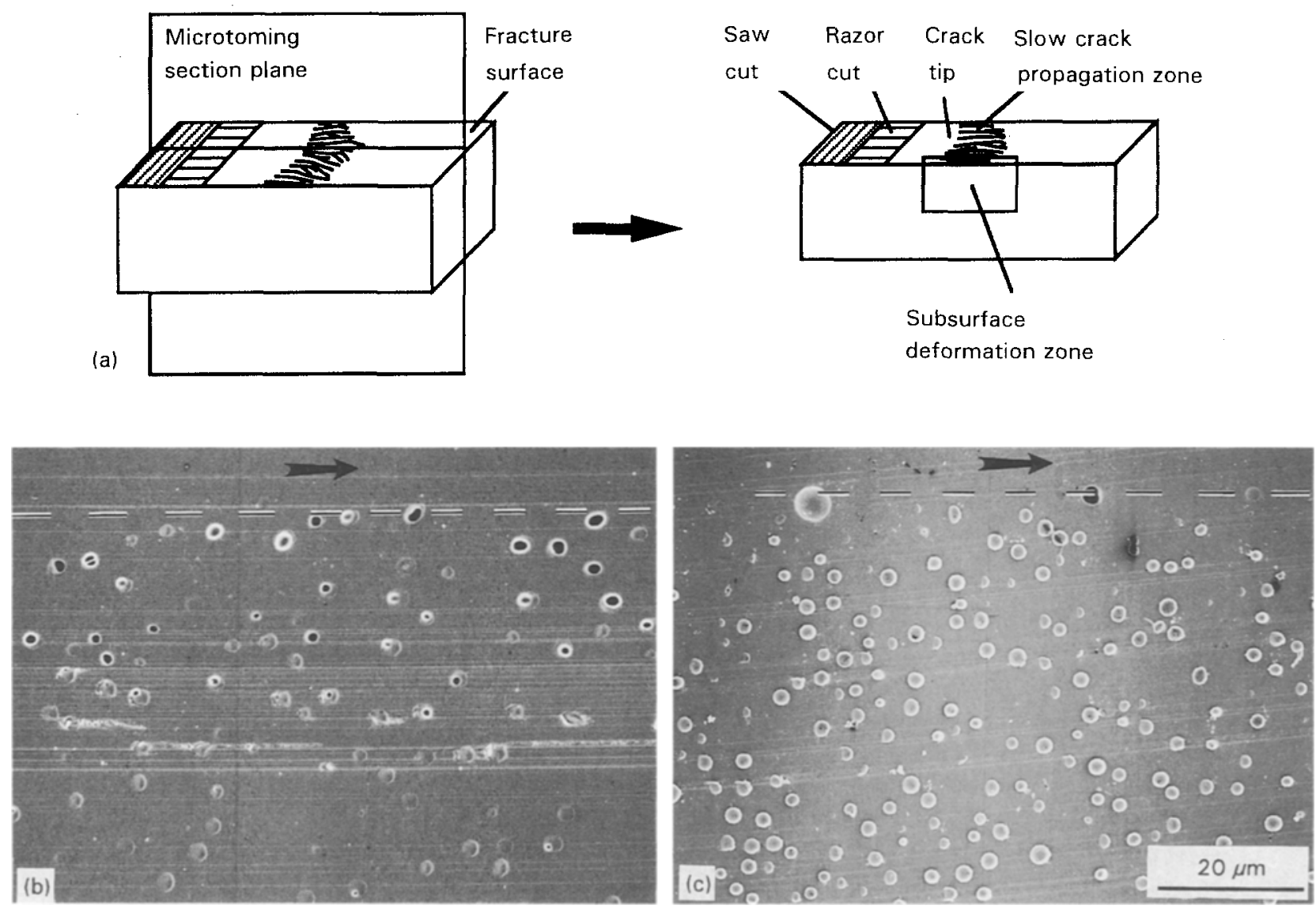

Figure 5 Preparation of microtomed section surfaces for SEM examination and the position micrographs taken are illustrated in (a) Micrographs of microtomed surfaces of CTBN-rubber-modified epoxy specimens tested at different pressures: (b) atmospheric pressure; (c) $80 \mathrm{MPa}$. Dashed lines in (b) and (c) denote the position of the fracture surface. Arrows indicate crack propagation direction. 


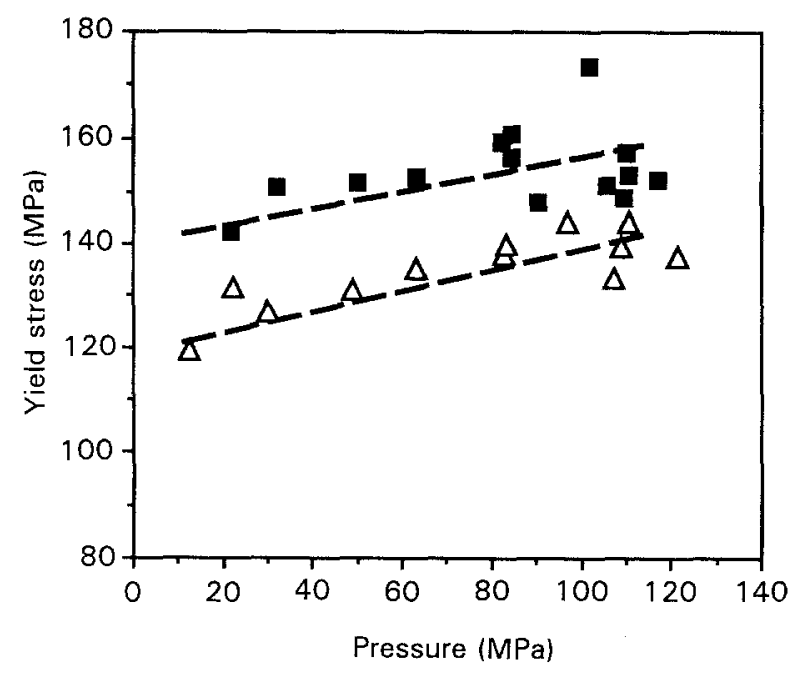

Figure 6 Tensile yield stress of $(\boldsymbol{\square})$ unmodified and $(\Delta) 10$ p.h.r. CTBN-rubber-modified epoxies versus hydrostatic pressure at $25^{\circ} \mathrm{C}$. Strain rate is $\sim 4 \mathrm{~s}^{-1}$.

increasing the superimposed hydrostatic pressure, crazing or cracking in blunt-notched bending specimens of PP, PE and PA-6 was suppressed or eliminated.

Another important point to note is that over the entire pressure range studied, the yield stress of CTBN-rubber-modified epoxy is lower than that of the unmodified epoxy. This indicates that, although rubber cavitation is suppressed by pressure, the CTBN-rubber still behaved like soft particles, which cause stress concentration and lower the tensile yield stress of the modified material. This fact is important to the discussion of the fracture toughness of unmodified and rubber-modified epoxy in the next section.

We also note that the superimposed hydrostatic pressure and high strain rate do not raise the glass transition temperature of the rubber to the point where it is near room temperature - the temperature range in which our tests were performed. According to Billinghurst and Tabor [33], the pressure coefficient of $T_{\mathrm{g}}$ is $0.18^{\circ} \mathrm{C} \mathrm{MPa}^{-1}$. Thus, at $80 \mathrm{MPa}$, the $T_{\mathrm{g}}$ of the rubber under pressure is $-41^{\circ} \mathrm{C}$. Changing the time scale by a factor of ten times will shift the apparent $T_{\mathrm{g}}$ by roughly $7^{\circ} \mathrm{C}$ for a typical polymer [34]. The strain rate in our test $\left(4 \mathrm{~s}^{-1}\right)$ is about 4000 times higher than the normal strain rate of $0.001 \mathrm{~s}^{-1}$. This strain-rate difference results in an increase of $25^{\circ} \mathrm{C}$ in $T_{\mathrm{g}}$. Assuming these two effects can be linearly combined, then the $T_{\mathrm{g}}$ of the rubber is $-16^{\circ} \mathrm{C}$, which is still below room temperature.

\subsection{The fracture toughness of unmodified and rubber-modified epoxies}

The dependence of the fracture toughness on pressure is shown in Fig. 7. The toughness of both unmodified and rubber-modified epoxies increases with pressure. The fracture toughness of 10 p.h.r. CTBN-rubbermodified epoxy under pressure is approximately $40 \%$ higher than that at atmospheric pressure, while the fracture toughness of the unmodified epoxy under

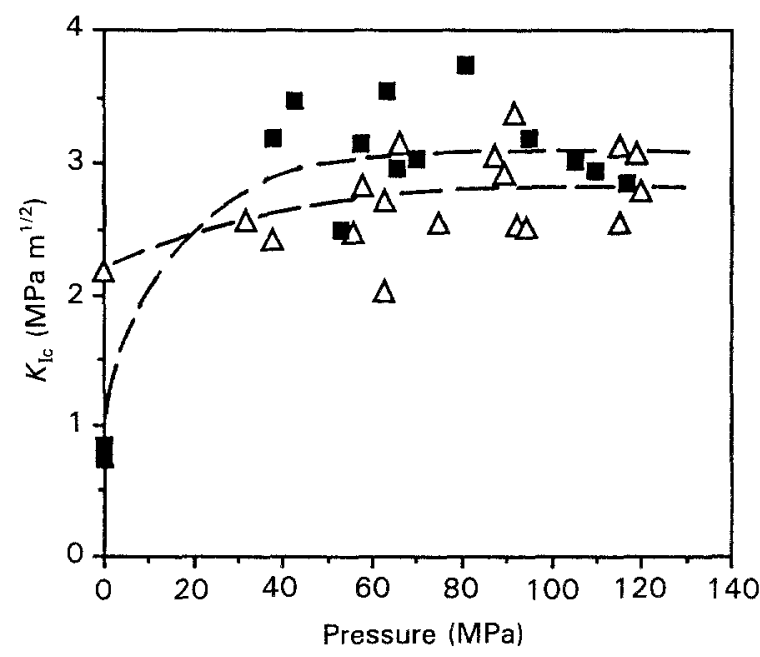

Figure 7 Fracture toughness of $(\boldsymbol{\square})$ unmodified and $(\Delta) 10$ p.h.r. CTBN-rubber-modified epoxies versus hydrostatic pressure at $25^{\circ} \mathrm{C}$.

pressure is approximately $300 \%$ higher than that under atmospheric pressure. The fracture toughness of the rubber-modified epoxy is no higher than that of the unmodified epoxy in the pressure range from 38-150 MPa. Unfortunately, no data are available for pressures below $38 \mathrm{MPa}$ due to the friction in the apparatus. Therefore, the pressure at which the toughness difference disappears for these two materials has not been determined.

\subsubsection{Why does neat epoxy become tougher under pressure?}

We will demonstrate in this section that near-surface plastic deformation enhanced by the superimposed hydrostatic pressure causes the toughness of the unmodified epoxy to increase. SEM was used to examine the fracture surfaces of unmodified epoxy, and the results are shown in Fig. 8. The fracture surface of an unmodified epoxy specimen tested at atmospheric pressure is shown in Fig. 8a. It is rather smooth and featureless. In contrast, at $60 \mathrm{MPa}$, the fracture surface near the starter crack is very rough (Fig. 8b). Fig. 8c and $d$ are the start and end regions of this rough zone at higher magnification. The roughness appears to be a result of plastic tearing of the material between crack planes initiated at different distances from the final fracture plane. To verify that this surface roughness is due to plastic deformation, a thin section perpendicular to the fracture surface and parallel to the direction of crack propagation was made in the middle of the specimen by petrographic polishing. The thin sections thus obtained were examined by optical microscopy and the results are shown in Fig. 9. Without crossed polarizers, the thin sections show the profile of the fracture surfaces. It is smooth and featureless in the epoxy specimen tested at atmospheric pressure (Fig. 9a), while that of the pressurized specimen has an elongated zone with a great deal of surface roughness (Fig. 9c). With crossed polarizers, the thin section from the pressurized specimen exhibits a very 

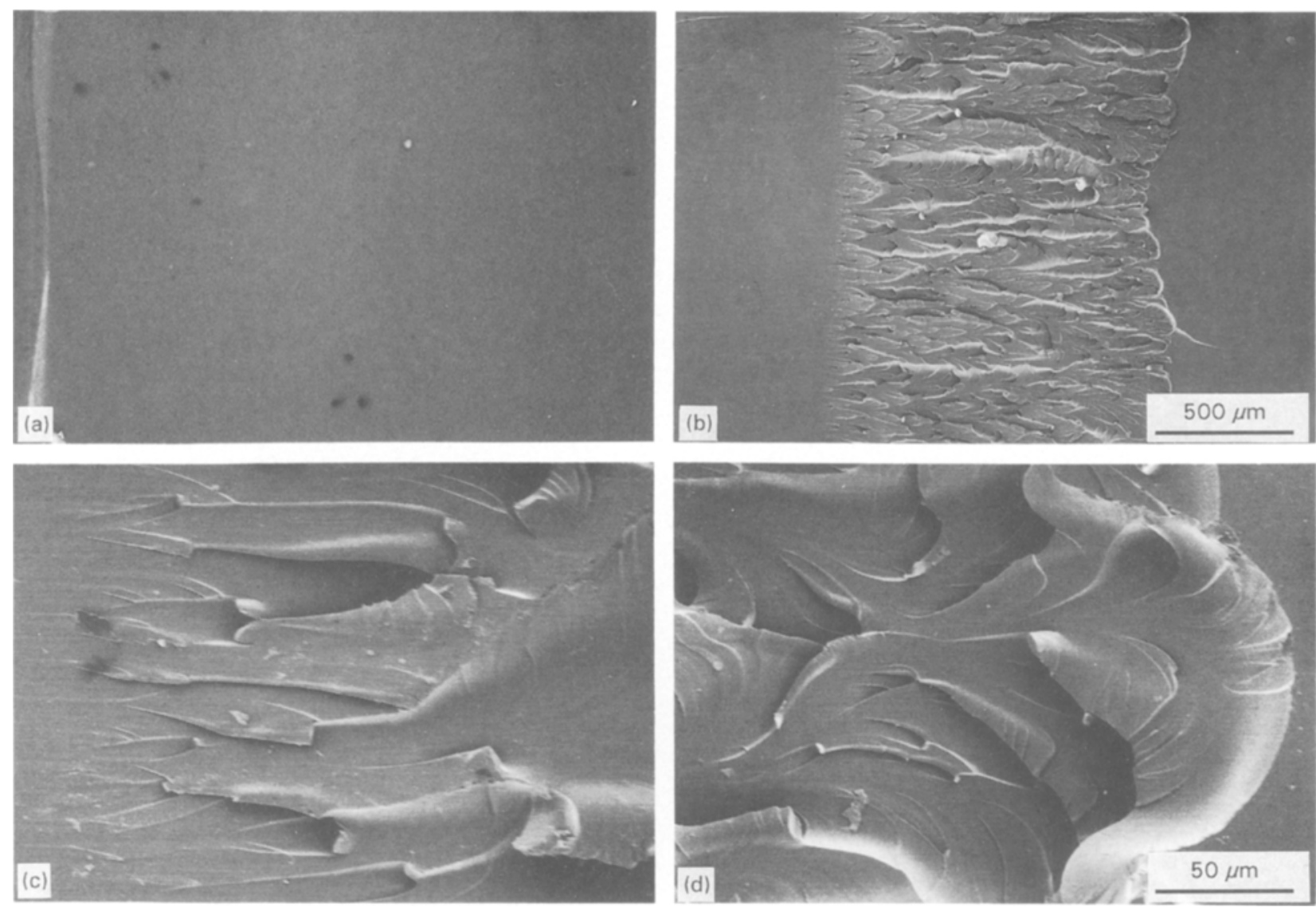

Figure 8 Scanning electron micrographs of the fracture surfaces of unmodified epoxy tested at different pressures: (a) atmospheric pressure; (b-d) $60 \mathrm{MPa}$.
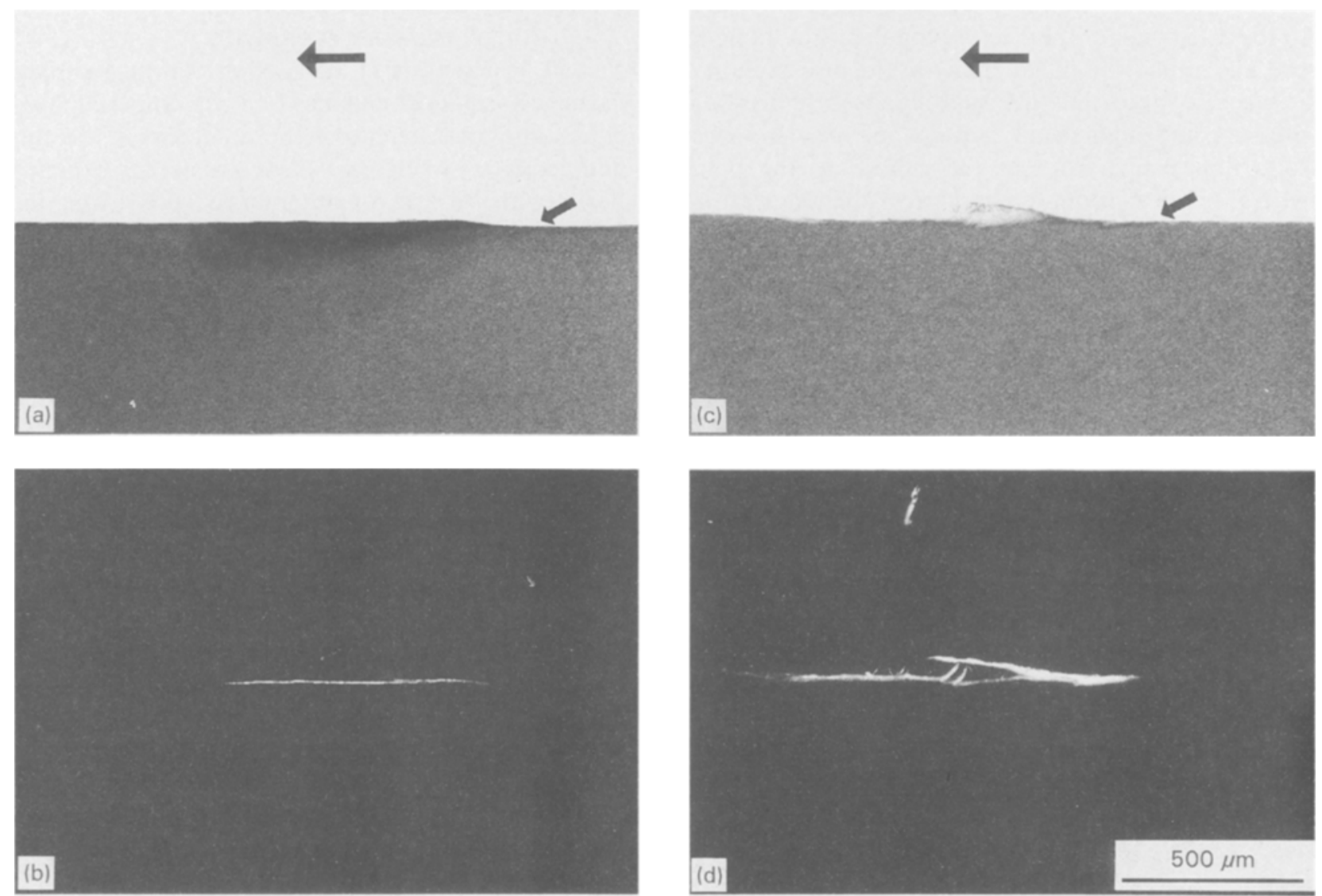

Figure 9 Optical micrographs of thin sections of unmodified epoxy tested at different pressures: (a, b) atmospheric pressure; (c, d) $60 \mathrm{MPa}$ The small arrow indicates the original crack tip position. The large arrow indicates the crack propagation direction. 
elongated birefringent zone (Fig. 9d), the result of plastic tearing near the surface. In contrast, the thin section from the specimen fractured at atmospheric pressure shows a very short and narrow birefringent zone (Fig. 9b), reflecting very little plastic deformation. From the comparison of the size and depth of the plastic birefringence zones, it is obvious that epoxy specimens tested under pressure would give much higher toughness values, which, in fact, they do.

We now turn to a discussion of why the amount of plastic tearing in unmodified epoxy is increased under pressure. The toughness of epoxy, like those of most amorphous polymer materials, is determined by the competition between the shear-deformation process and the cracking process. (Because the occurrence of crazing has not been confirmed in epoxies, we refer to the cracking process only.) These two processes are both strongly affected by the hydrostatic pressure, but their pressure dependences are different. If the maximum deviatoric stress in front of the sharp crack reaches the critical value for shear-yielding first, then the specimen will yield and undergo shear deformation. On the other hand, if the maximum mean stress reaches the critical value for cracking first, then the specimen will fail by cracking, with little shear deformation. At atmospheric pressure, epoxy specimens with a sharp crack are brittle because the cracking process caused by high mean stress is preferred, and they fail with very little plastic deformation (see Fig. 9a and c). With the superimposed hydrostatic pressure, the tensile mean stress is substantially lowered. As a result, cracking is postponed to a later stage. When cracking does eventually occur it appears to be nucleated from many sites (Fig. 8b) and more plastic tearing can occur. This results in a larger plastic zone (Fig. 9b and d) and leads to a large increase in the fracture toughness. We hypothesize that the roughness is due to the nucleation of many cracks at almost the same stress or strain level. Because of the plastic stability and the postponement of the cracking process by pressure, more cracks can be nucleated. When these cracks merge, the material between approaching crack fronts tears plastically.

The pressure dependence of the fracture toughness of epoxy is quite different from that of $\mathrm{PE}$ found by Duckett and co-workers $[23,24]$. The reason for this is that PE is a ductile polymer, and its fracture mode at atmospheric pressure is shear deformation dominant. With increasing pressure, the amount of plastic deformation is reduced due to increased yield stress so that the toughness decreases. The mode change is from ductile to brittle. In the epoxy resins we used at atmospheric pressure the brittle cracking process is in control. With applied hydrostatic pressure, the cracking process is postponed, thus allowing some plastic deformation to occur, increasing the fracture toughness rather than decreasing it. Furthermore, the pressure range we studied (up to $150 \mathrm{MPa}$ ) is much smaller than that studied by Duckett and co-workers (up to $700 \mathrm{MPa}$ ). In our epoxy system we expect that as the hydrostatic pressure increases beyond a certain value, the yielding process will become more difficult and the toughness may drop.

\subsubsection{The toughness increase in rubber- modified epoxy under pressure}

Optical and scanning electron microscopy reveal that, similar to the case of the unmodified epoxy mentioned above, plastic deformation also causes toughness to increase in CTBN-rubber-modified epoxy. As shown in Fig. 4, the surface of a CTBN-rubber-modified epoxy specimen fractured under hydrostatic pressure has a larger plastic tearing zone than that of a specimen fractured at atmospheric pressure, even though the cavitation of CTBN-rubber has been suppressed. The fracture surface of the rubber-modified epoxy under pressure is more similar to that of the unmodified epoxy fractured under high pressure (Fig. 8) than to that of the rubber-modified epoxy fractured under atmospheric pressure (Fig. 4a and b). A specimen fractured at atmospheric pressure has a cavitation zone that scatters light strongly (Fig. 10a), but one fractured under high pressure $(80 \mathrm{MPa})$ does not (Fig. 10c). With crossed polarizers, the birefringent zone of the rubber-modified epoxy specimen fractured at atmospheric pressure (Fig. 10b) is larger than that of an unmodified epoxy specimen fractured at the same pressure (Fig. 9b), exhibiting enhanced plastic deformation due to cavitation [8]. However, the size of this subsurface zone is smaller than the plastic tearing zone of a rubber-modified epoxy specimen fractured under pressure (Fig. 10d). These observations are the results of two different mechanisms which both reduce the mean stress. The cavitation of CTBN-rubber particles provides an internal mechanism for the relief of mean stress, while hydrostatic pressure provides an external mechanism for the reduction of mean tensile stress. Both mechanisms result in an increase in fracture toughness. The external pressure increases toughness by increasing the amount of plastic tearing near the surface, while the cavitation increases the amount of plastic flow via both plastic dilatation and shear localization. When the pressure is increased the material switches from one fracture mechanism to the other. Thus, by applying a hydrostatic pressure which suppresses cavitation, a slight net increase of surface plastic deformation and hence toughness, compared to unpressurized state, results.

\subsubsection{Why is the fracture toughness of rubber-modified epoxy no higher than that of neat epoxy?}

When rubber cavitation is suppressed by superimposed hydrostatic pressure, the fracture toughness of CTBN-rubber-modified epoxy is no higher than that of the unmodified epoxy (Fig. 7). Scanning electron micrographs of the fracture surface show that, with identical geometries and crack lengths, the size of the rough zone on the fracture surface of 10 p.h.r. CTBNrubber-modified epoxy (Fig. 4c, d) is smaller than that of the unmodified epoxy (Fig. 9b-d), while the degree of roughness is comparable. The deformation giving rise to the roughness in these zones is plastic tearing. However, there is less plastic tearing in the rubbermodified epoxy than in the unmodified epoxy. On the basis of the above observations, and the facts that the 

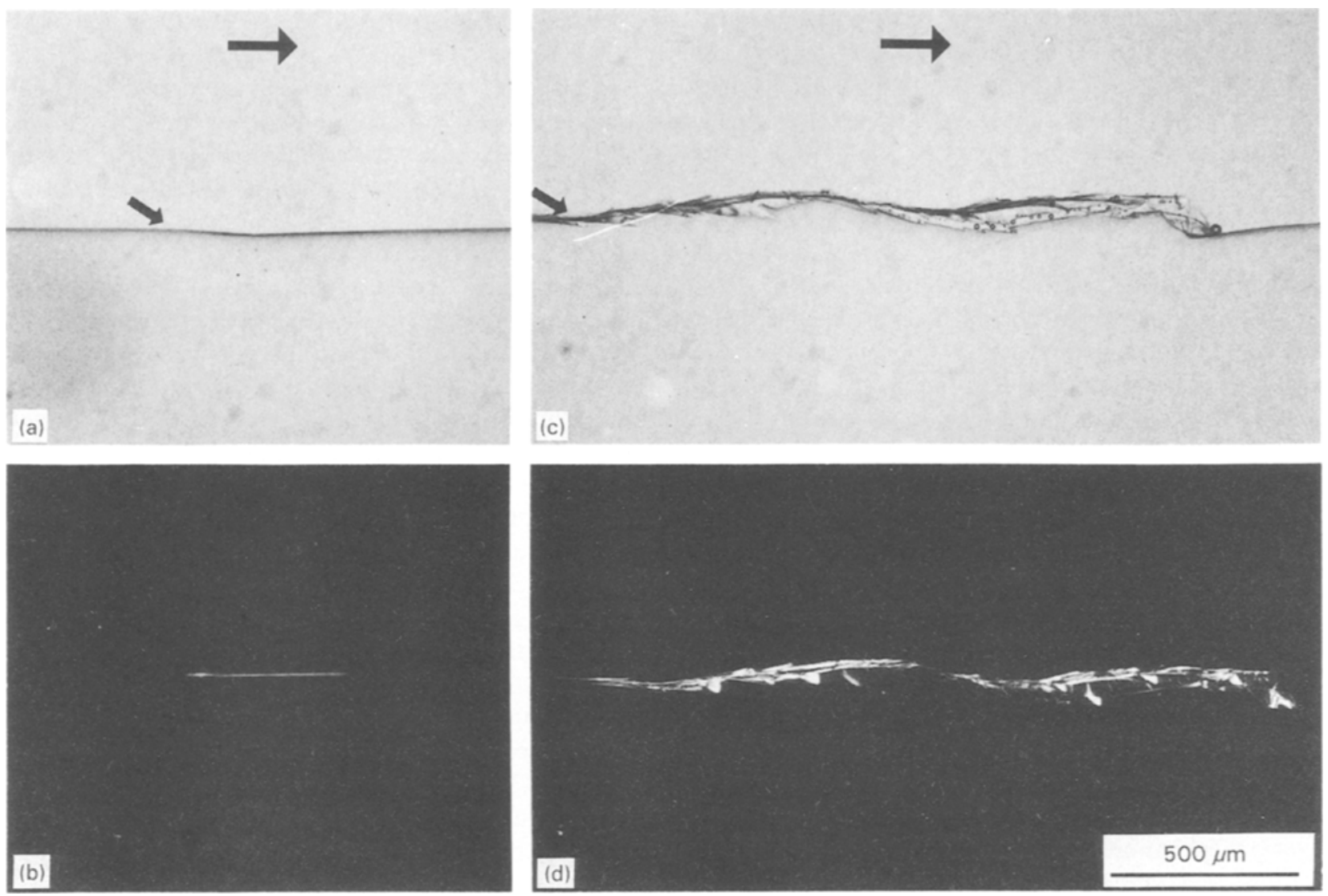

Figure 10 Optical micrographs of thin sections of CTBN-rubber modified epoxy tested at different pressures: (a, b) atmospheric pressure; (c, d) $80 \mathrm{MPa}$. The small arrow indicates the original crack tip position. The large arrow indicates the crack propagation direction.

epoxy becomes more ductile under hydrostatic pressure and that CTBN-rubber cavitation is prevented by pressure, we conclude that a CTBN-rubber that is incapable of cavitation does not increase the fracture toughness of an epoxy matrix which already possesses the capability for shear plasticity. This implies that (1) stress concentration by rubber-particles alone will not necessarily induce massive shear-yielding and increase the fracture toughness, and (2) cavitation is very important to the toughening. Without cavitation, even though these rubber particles can still cause stress concentration, they are not effective in toughening. They may actually decrease the fracture toughness somewhat, perhaps due to their low cohesive strength and shear strength, although such a decrease cannot be ascertained given the large scatter in the data obtained (Fig. 7).

\subsubsection{Final remarks}

In the discussions above, we do not attempt to quantify the contribution of dilatational plasticity to the total fracture toughness in rubber-modified epoxies because this complicated subject involves other critical aspects of the theory of toughening of many plastics, and is best dealt with elsewhere $[35,36]$. We note, however, that attempts to partition energyabsorbing mechanisms into various processes as if they are linearly additive miss, perhaps, the most important conclusion reached in this paper, namely, cavitation provides the key to effective toughening by the rubber particles. It is the enabling event. Other subsequent events also have a causal relationship when the volume fraction of rubber particles is low, i.e. plastic dilatation increases the effective volume fraction of voids, which interact non-linearly to form localized shear bands. These sequential, interacting events produce a total level of toughness far greater than the sum of these events acting independently, if they are able to occur. Although, in the present case, another mechanism (plastic tearing) takes over when cavitation is suppressed, this beneficial effect is more the result of pressure-induced suppression of the cohesive failure of the matrix. Put another way: if the rubber particles are prevented from cavitating by heavy cross-linking, then the toughness would decrease, this time without the extra plastic tearing to compensate for the loss of the sub-surface plastic shear mechanisms [37].

One other point worth noting is the pressure required to suppress dilatational plasticity. According to a theory by Andrews and Bevan [38], the net hydrostatic tension $D$ necessary to plastically enlarge a cavity in an elastic-plastic solid is $D=\Psi \sigma_{y}$ if the surface energy term is neglected for cavities of this size. $\Psi$, the propagation constant, is between 2.4 and 3.3 for a wide range of plastics. Thus, to suppress plastic dilatation, the external pressure must be increased to over $300 \mathrm{MPa}$, which is about ten times the critical pressure found. This observation provides further clear evidence for the critical role played by the cavitation of the particles. By suppressing this one event, the plastic dilatation cannot occur at all in the given stress state. 


\section{Conclusions}

The tensile properties and fracture toughness of unmodified and CTBN-rubber-modified epoxies was studied under hydrostatic pressure. The underlying mechanisms responsible for the property changes as a result of pressure were analysed by a combination of optical and SEM microscopy. Our findings are as follows.

1. The unmodified epoxy exhibits a brittle-toductile transition in both tensile and fracture toughness tests. The reason for this transition is the postponement of the cracking process by the applied pressure.

2. Cavitation of CTBN-rubber can be suppressed at a relatively low pressure. For the current material system, the minimum pressure required to eliminate CTBN-rubber cavitation is between 30 and $38 \mathrm{MPa}$.

3 . With cavitation suppressed, the rubber particles are unable to induce massive shear-yielding in the epoxy matrix, and the fracture toughness of the rubber-modified epoxy is derived from surface tearing just like that in unmodified epoxy.

\section{Acknowledgements}

The work performed at the University of Michigan was supported by an NSF MRG grant, no. 8708405 , and a NIH NIDR grant to the Specialized Materials Center in the School of Dentistry. The work performed at Kyushu University was supported by a grant from Monbushu of Japan to facilitate scientific exchange between the research groups of Professor K. Takahashi and Professor A. F. Yee. The assistance of Mr Yashuhilo Sakurada in setting up the pressure apparatus is gratefully acknowledged.

\section{References}

1. W. D. BASCOM and D. L. HUNSTON, in "Adhesion 6", edited by K. W. Allen (Applied Science, London, 1980).

2. D. L. HUNSTON and W. D. BASCOM, ACS Adv. Chem. Ser. 208 (1984) 83.

3. A. F. YEE and R. A. PEARSON, J. Mater. Sci. 21 (1986) 2462.

4. R. A. PEARSON and A. F. YEE, ibid. 21 (1986) 2475.

5. A. J. KINLOCH, S. J. SHAW, D. A. TOD and D. L. HUNSTON, Polymer 24 (1983) 1341.

6. A. J. KINLOCH, S. J. SHAW and D. L. HUNSTON, ibid. 24 (1983) 1356.

7. R. A. PEARSON and A. F. YEE, J. Mater. Sci. 26 (1991) 3828.

8. A. F. YEE, DONGMING LI and XIAOWEI LI, ibid, submitted.
9. A. NADAI, Z. Physik. 30 (1924) 115.

10. P. W. BRIDGMAN, "Studies in Large Plastic Flow and Fracture" (McGraw-Hill, New York, 1952).

11. K. MATSUSHIGE, S. V. RADCLIFFE and E. BAER, J. Mater. Sci. 10 (1975) 833.

12. L. HOLliday, J. MANN, G. A. POGANY, H. PUGH and D. A. GUNN, Nature 202 (1964) 381.

13. H. PUGH, E. F. CHANDLER, L. HOLLIDAY and J. MANN, Polym. Eng. Sci. 11 (1971) 463.

14. G. G. BIGLIONE, E. BAER and S. V. RADCLIFFE, in "Proceedings of the Second International Conference on Fracture", Brighton, 1969, edited by P. L. Pratt (Chapman and Hall, London, 1969).

15. K. MA TSUShige, J. Polym. Sci. Polym. Phys. 14 (1976) 703.

16. S. RABINOWITZ and I. M. WARD, J. Mater. Sci. 5 (1970) 29.

17. J. S. HARRIS, I. M. WARD and J. S. C. PARRY, J. Mater. Sci. 6 (1971) 110.

18. J. YUAN, A. HILTNER and E. BAER, ibid. 18 (1983) 3063

19. S. K. BHATEJA and K. D. PAE, Polym. Lett. 10 (1972) 531.

20. A. MOET and E. BAER, J. Mater. Sci. 15 (1980) 31.

21. J. S. TRENT, M. J. MILES and E. BAER, ibid. 14 (1979) 789

22. R. A. DUCKET T, ibid. 15 (1980) 2471.

23. R. W. TRUSS, R. A. DUCKETT and I. M. WARD, ibid. 19 (1984) 413.

24. J. SWEENEY, R. A. DUCKETT and I. M. WARD, J. Mater. Sci. Lett. 5 (1986) 1109.

25. F. J. GUILD and R. J. YOUNG, J. Mater. Sci. 24 (1989) 2454

26. E. J. DEKKERS, S. Y. HOBBS and V. H. WATKINS, ibid. 23 (1988) 1225.

27. C. K. RIEW, E. H. ROWE and A. R. SIEBERT, $A C S A d v$. Chem. Ser. 154 (1976) 326.

28. K. TAKAHASHI and T. OGIHARA, J. Soc. Mater. Sci. Jpn 40 (1991) 185.

29. J. AGRANOFF (ed.), "Modern Plastics Encyclopedia" (McGraw-Hill, New York, 1991).

30. J. A. SAUER, K, D. PAE and S. K. BHATEJA, J. Macromol. Sci. B8 (1973) 631.

31. M. ISHIKAWA, I. NARISAWA and H, OGAWA, J. Polym. Sci. 15 (1977) 1791.

32. I. NARISAWA and M. ISHIKAWA, in "Advances in Polymer Science", Vol. 91/92, edited by H.-H. Kausch (Springer, New York, 1990) p. 353.

33. P. R. BiLLINGHURST and D. TABOR, Polymer 12 (1971) 101.

34. L. E. NIELSEN, "Mechanical Properties of Polymers and Composites", Vol. 1 (Marcel Dekker, New York, 1974) p. 19.

35. D. LI, X. LI and A. F. YEE, Proc. ACS Polym. Mater. Sci. Eng. 63 (1990) 296.

36. D. LI and A. F. YEE, J. Mater. Sci., submitted.

37. D. LI, PhD thesis, University of Michigan (1993).

38. E. H. ANDREWS and L. BEVAN, Polymer 13 (1972) 337.

Received 4 May

and accepted 18 August 1993 